
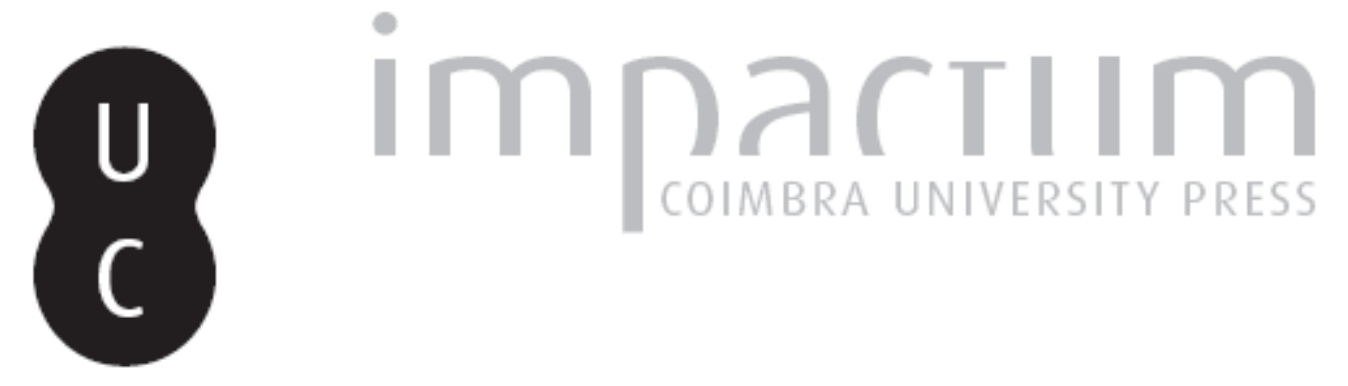

\title{
Prove di liberalismo: Maria Pia e Umberto di Savoia nel Portogallo del 1862
}

Autor(es): $\quad$ Mancusa, Mauro la

Publicado por: Imprensa da Universidade de Coimbra

URL persistente:

URI:http://hdl.handle.net/10316.2/42554

DOI:

DOI:https://doi.org/10.14195/0870-8584_6_11

Accessed : $\quad$ 26-Apr-2023 15:23:54

A navegação consulta e descarregamento dos títulos inseridos nas Bibliotecas Digitais UC Digitalis, UC Pombalina e UC Impactum, pressupõem a aceitação plena e sem reservas dos Termos e Condições de Uso destas Bibliotecas Digitais, disponíveis em https://digitalis.uc.pt/pt-pt/termos.

Conforme exposto nos referidos Termos e Condições de Uso, o descarregamento de títulos de acesso restrito requer uma licença válida de autorização devendo o utilizador aceder ao(s) documento(s) a partir de um endereço de IP da instituição detentora da supramencionada licença.

Ao utilizador é apenas permitido o descarregamento para uso pessoal, pelo que o emprego do(s) título(s) descarregado(s) para outro fim, designadamente comercial, carece de autorização do respetivo autor ou editor da obra.

Na medida em que todas as obras da UC Digitalis se encontram protegidas pelo Código do Direito de Autor e Direitos Conexos e demais legislação aplicável, toda a cópia, parcial ou total, deste documento, nos casos em que é legalmente admitida, deverá conter ou fazer-se acompanhar por este aviso.

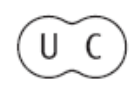




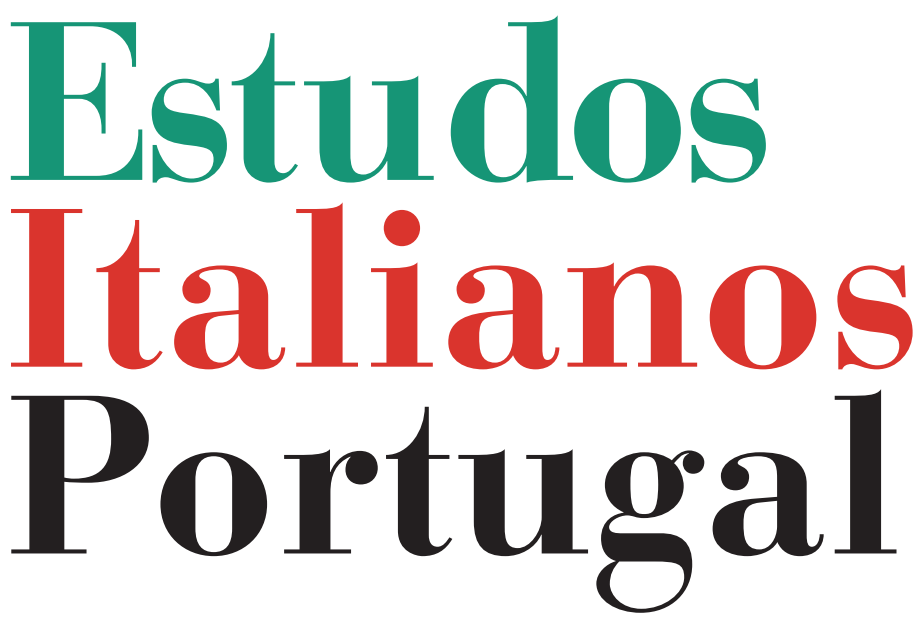

Instituto

Italiano

de Cultura

de Lisboa

Nova Série

$\mathrm{N}^{\circ} 6$ 
Per il Portogallo, il 1861 si era chiuso tragicamente. L'11 novembre moriva, a soli ventiquattro anni, il re D. Pedro V, vittima di una febbre tifoide contratta durante un soggiorno di caccia in Alentejo. Contagiati dallo stesso male morivano anche i fratelli D. João e D. Fernando. Di cinque principi, rimanevano in vita soltanto D. Luís e D. Augusto (che pure era stato contagiato, ma riuscì a sopravvivere). Nonostante la diagnosi ufficiale, a palazzo e per le strade correvano ipotesi di avvelenamento "que o vulgo acreditou facilmente" e che diedero origine ai tumultos de Natal'. Era in queste circostanze che D. Luís I, a soli ventitré anni, diventava re del Portogallo. Il nuovo re non era sposato. Il fratello era un adolescente e non si era ripreso del tutto dal contagio. Riappariva, così, lo spettro della crisi dinastica. A tal proposito, il parlamento approvò alcune leggi per regolare la successione in caso di morte senza eredi del sovrano, che a sua volta si affrettò a prendere moglie per assicurare la discendenza.

* Mauro La Mancusa (Messina, 1988) ha lavorato in quanto stagista del programma Erasmus placement, stabilito tra l'Università di Pisa e l'Università di Coimbra, presso l'Istituto di Studi Italiani di questa Università. Si occupa dei rapporti culturali tra Portogallo e Italia nell'Ottocento.

${ }^{1}$ Damião Peres, História de Portugal, Barcelos, Portucalense/Porto, Livraria Civilização, vol. 7, pp. 357-360. 
Il 1862 si apriva dunque con la ricerca di una regina. Ricerca in cui si incrociarono, com'era consueto, fattori di diversa natura, finché la scelta non ricadde sulla quindicenne Maria Pia di Savoia. La candidatura della principessa piemontese era stata caldeggiata fin da subito dal visconte di Sá de Bandeira, ministro della guerra nel governo del partito histórico di Loulé. A suo dire, rispetto alle altre opzioni, dall'apporto politico nullo o addirittura controproducente, la scelta di Maria Pia prometteva più di un beneficio. Innanzitutto, i Savoia godevano di grande popolarità tra i liberali portoghesi, che avevano seguito con entusiasmo l'impresa dei Mille e avevano fatto proprio, come vedremo anche più avanti, il mito del Risorgimento. Inoltre, il neonato Regno d'Italia appariva come un alleato prezioso per i destini del Portogallo negli equilibri internazionali. Sá da Bandeira, peccando forse di ottimismo, contava sulla reputazione del neonato Regno d'Italia presso le grandi potenze europee in vista di future dispute coloniali. Tuttavia la questione era ben lungi dall'essere chiusa. Se da un lato i Savoia erano ben visti dal governo liberale, non mancavano pareri contrari, come quello della regina Vittoria, motivato dalla giovanissima età della principessa e, più verosimilmente, dalla politica di Vittorio Emanuele II (e di Napoleone III). Il suo parere, oltre a smentire in parte Sá da Bandeira riguardo alla reputazione dei Savoia, dovette impressionare abbastanza D. Luís I, il quale seguì il consiglio della regina e chiese la mano di Teresa, arciduchessa d'Austria. Infine, in seguito al rifiuto dell'arciduca, si decise per Maria Pia e ne chiese e ottenne la mano, con buona pace della regina Vittoria. Questa non poté fare altro che commentare il fatto compiuto e rinfacciare al giovane re il brusco ripensamento ${ }^{2}$.

${ }^{2}$ Luís Nuno Espinha da Silveira, Paulo Jorge Fernandes, D. Luís, Lisboa, Círculo de Leitores, 2006, pp. 37-42. 
Il 4 settembre 1862, convocate le Cortes Gerais Extraordinárias, D. Luís I annunciava alle camere la propria decisione, sottolineando che l'unione con la principessa piemontese rinnovava "uma aliança que data da fundação

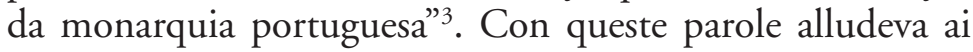
numerosi legami matrimoniali tra dinastie lusitane e casa di Savoia, che erano iniziati addirittura nel XII secolo con le nozze tra Mafalda di Savoia e Afonso Henriques, fondatore appunto della monarchia portoghese. I precedenti illustri del matrimonio furono ricordati anche da numerosi articoli, saluti e pubblicazioni celebrative che accompagnarono il periodo delle nozze.

Ancor prima dell'annuncio ufficiale, le voci sul matrimonio tra D. Luís e Maria Pia avevano ravvivato il dibattito sulle vicende italiane, che già da qualche tempo infiammava la stampa e l'opinione pubblica portoghese. Se, da un lato, il Portogallo liberale aveva fatto proprio il mito dell'Italia redimida, dall'altro la stampa cattolica e conservatrice aveva ingaggiato una battaglia contro Vittorio Emanuele II, colpevole di attentare al potere temporale del papato. A Nação, giornale cattolico e conservatore, con toni apocalittici e grande abbondanza di punti esclamativi, ritraeva l'Unità d'Italia come un'autentica rivoluzione che si sarebbe fermata solo con la "conversão da portentosa basílica de S. Pedro de Roma em templo da Deusa Razão", e temeva la componente radicale del Risorgimento al punto da sostenere che "a revolução italiana é Mazzini e Garibaldi, a inteligência e o braço: o governo de Turim não é senão um acessório" ${ }^{\text {. Non }}$ va dimenticato che proprio nell'estate del 1862 l'iniziativa di Garibaldi e la giornata dell'Aspromonte avevano riproposto prepotentemente la questione romana anche in Portogallo. Date le premesse, è facile immaginare il parere di $A$ Nação

${ }^{3}$ Diário de Lisboa, n. ${ }^{\circ} 200,5-9-1862$.

${ }^{4}$ A Nação, 16-10-1862. 
riguardo alla scelta di Maria Pia: "A princesa escolhida é filha dum excomungado. Isto diz tudo"s.

Ma c'è dell'altro. Agli occhi di $A$ Nação, la popolarità presso i liberali portoghesi della casa di Savoia e del Risorgimento assumeva un significato che andava ben oltre la questione romana e riguardava un problema più specificamente portoghese: la questione dell'iberismo. Nella seconda metà dell'ottocento era tornata d'attualità l'idea della fusione con la Spagna, articolata in forme molto diverse (dall'unione dinastica al federalismo repubblicano, senza dimenticare l'internazionalismo socialista e altre utopie ecumeniche) ${ }^{6}$. A quest'idea si opponeva un deciso fronte nazionalista e antiiberista, di cui faceva parte anche $A$ Nação: "Que política é pois a que Portugal adopta com o Piemonte? Será a tão preconizada política das grandes monarquias? Será a da usurpação e roubo dos soberanos vizinhos, e da usurpação sacrílega dos Estados da Igreja?" e ancora: "O iberismo tem tomado diversas cores" . Agli occhi di un giornale come A Nação, le annessioni dei Savoia erano una minaccia diretta per il Vaticano e indiretta per il Portogallo, in quanto la politica delle annessioni avrebbe potuto servire d'esempio ai liberali portoghesi. Questi ultimi, infine, venivano accusati di fomentare un iberismo monarchico solo temporaneamente, per poi dedicarsi a un più sovversivo iberismo repubblicano.

Di conseguenza, non sorprende la scelta dello stesso giornale di boicottare le nozze e le celebrazioni, limitandosi a un articoletto di poche righe: "Chegou ontem pelo meio dia à barra do Tejo a flotilha, que fora a Genova. Vinha acompanhada de quatro fragatas sardas e um vapor pequeno. Os festejos prosseguem segundo o programa. O povo vê e procura

${ }^{5}$ A Nação, 15-7-1862.

${ }^{6}$ Fernando Catroga, Nacionalistas e iberistas, História de Portugal, ed. José Mattoso, Lisboa, Estampa, s.d., vol. 5, pp. 563-567.

${ }^{7}$ A Nação, 23-10-1862. 
ver com curiosidade que é natural, e nada mais" ${ }^{\text {. }}$. Il tono dell'articolo colpisce, se si pensa che nelle stesse settimane "toda a imprensa liberal de Lisboa saúda hoje por um modo distintíssimo o régio enlace, e enche as suas colunas com a descripção dos festejos com que a capital da monarquia tomou singular parte nesse solene acto" ". Dai giornali liberali, infatti, abbiamo un resoconto dettagliato delle nozze e dei festeggiamenti. Il 20 settembre arrivava a Genova, con la flotilha portoghese, il primo ministro Loulé in qualità di plenipotenziario portoghese. Il 27 settembre, nella cappella reale di Torino, aveva luogo il matrimonio per procura. Il $28 \mathrm{Ma-}$ ria Pia salpava per il Portogallo, accompagnata dal fratello, il principe Umberto. Il 5 ottobre la flotta italo-portoghese entrava nel Tago e il 6 ottobre nella chiesa di San Domenico avveniva la ratifica del matrimonio.

Mentre a Lisbona si festeggiavano le nozze e il compleanno della regina, a Coimbra e a Oporto fervevano i preparativi per la visita del principe Umberto. Se la visita ad Oporto, ultima dimora di Carlo Alberto, era annunciata come "um dever pio e sagrado à memoria do seu augusto avô" 10 , ben più importante si rivelò il passaggio da Coimbra, per una serie di circostanze di cui il giovane principe doveva sapere poco o nulla.

Pinto Osório racconta che il principe era accompagnato nel suo viaggio da un certo "Miguel de Sá Nogueira, rapaz muito gentil, um dos estudantes (chamava-se o outro João Ferrão Castelo Branco) que, dois anos antes, haviam ido bater-se pela causa da independência e da unidade de Itália, entrando em fogo na batalha de Solferino"11. Allo stesso episodio allude un articolo italiano, tradotto dal gior-

${ }^{8}$ A Nação, 6-10-1862.

${ }^{9}$ Diário de Lisboa, 9-10-1862.

${ }^{10}$ Diário de Lisboa, 23-10-1862.

${ }^{11}$ Pinto Osório, Lembranças da mocidade, Porto, Empresa Literária e Tipográfica, 1907, p. 287. 
nale portoghese $A$ Opinião e riportato dal Diário de Lisboa, in cui si legge che il giovane portoghese in realtà non aveva fatto in tempo a battersi contro gli austriaci, essendo arrivato subito dopo l'armistizio di Villafranca ${ }^{12}$. L'adesione di un giovane portoghese alla battaglia per l'indipendenza e per l'unità d'Italia è presentata da entrambe le fonti come una dimostrazione della solidariedade che i portoghesi, e in particolare gli studenti di Coimbra, provavano nei confronti dei popoli oppressi come la Polonia e, appunto, l'Italia ${ }^{13}$.

In effetti, l'attenzione alle vicende italiane caratterizza gli studenti di Coimbra prima, durante e dopo il 1862. Più che attenzione, è lecito parlare di entusiasmo, parola che ricorre costantemente nei memoriali. Infatti, se Eça de Queiroz parla di "largos entusiasmos europeus que logo adoptávamos como nossos, o culto de Garibaldi e da Itália redimida, a violenta compaixão da Polónia retalhada, o amor à Irlanda, a verde Erin, a esmeralda céltica, mãe dos Santos e dos Bardos, pisada pelo Saxónio!" ${ }^{4}$, Mariano Machado de Faria e Maia racconta che "Para aviventar o nosso ardor revolucionário, concorria a leitura assídua das obras de Proudhon, de Victor Hugo, de Daniel Stern, e mais que tudo o entusiasmo que sentíamos pela epopeia italiana, de que eram protagonistas Garibaldi e os seus camisas vermelhas" ${ }^{15}$. Infine, secondo João Machado de Faria e Maia, "todas as simpatias de Antero

12 "Entusiasta pela causa italiana, veio de Lisboa na época da última guerra da nossa independência contra a Áustria, com a intenção de tomar parte na nossa luta, porém chegou quando acabava de ser assignado o tratado de Villafranca”. Curiosamente, nello stesso articolo si legge che Sá Nogueira era nipote del visconte di Sá de Bandeira, che tanto aveva sostenuto la scelta di Maria Pia (Diário de Lisboa, 13-11-1862).

13 "Houve dos denodados rapazes, que abandonaram os seus estudos para ir bater-se pela emancipação da Itália! E lá se bateram na batalha de Solferino! Era assim que então a mocidade compreendia e executava a solidariedade!" (Pinto Osório, Lembranças da mocidade, p. 25).

${ }^{14}$ Antero de Quental, In memoriam, Porto, Mathieu Lugan, 1896, p. 485.

${ }^{15}$ Ib., p. 431. 
e da mocidade daquele tempo, entusiasticamente garibaldina, em relação às indecisas questôes italianas, convergiam assim naturalmente para a união e acção contra a propaganda autoritária, religiosa e catedrática, que em toda a parte procurava suscitar os mais decididos embaraços à unidade da Itália" ${ }^{16}$, ovvero proprio la propaganda di $A$ Nação. Lo stesso entusiasmo per l'Italia si ritrova in Antero de Quental, studente a Coimbra dal 1858 al 1864 . Oltre a conoscere ed ammirare la cultura e la letteratura del nostro paese, Antero ne seguiva le vicende politiche e militari, a cui si riferiscono varie poesie (À Itália, A Gennaro Perrelli), prose politiche ( $A$ Questão romana, A defesa da Carta Encíclica de Sua Santidade Pio IX) e due lettere, corrispondenti a due avventurosi progetti di viaggio in Italia ${ }^{17}$.

Di questo mito risorgimentale a Coimbra è interessante ricercare le cause e, soprattutto, le conseguenze nella vita studentesca. Secondo Eça de Queiroz è evidente che gli studenti solidarizzassero con i polacchi, con gli italiani e con gli irlandesi poiché, come loro, si sentivano oppressi da un tiranno, il rettore dell'Università Basílio Alberto de Sousa Teles ${ }^{18}$. Ne

${ }^{16} \mathrm{Ib}$., p. 150.

${ }^{17}$ Nella prima, del 1866, Antero invitava l'amico António de Azevedo Castelo Branco ad arruolarsi insieme a lui nei garibaldini. Nella seconda, del 1868, esortava Alberto Sampaio ad entrare addirittura nella guarda pontificia (Antero de Quental, Cartas, ed. Ana Maria Almeida Martins, Lisboa, IN-CM, 2009, pp. 14-16, 109-110, 159-160).

${ }^{18}$ Basílio Alberto de Sousa Teles fu rettore dell'Università di Coimbra tra il 1859 e il 1863. Malgrado il passato da combattente liberale, attirò fin da subito le critiche degli studenti per il conservatorismo formale e per la severità delle misure disciplinari. Tuttavia, la protesta della Sociedade do Raio va ben oltre la persona del rettore e s'inserisce nel contesto più ampio del dibattito per la laicizzazione e modernizzazione dell'università di Coimbra, che sembrava incompatibile con le nuove istituzioni liberali. Scrive Teófilo Braga: "Para resistir a todas as modificações do meio social desde o fim do século XII até ao fim do século XIX, a Universidade enquistou-se na sua Tradição: os hábitos clericais, as suas cerimónias ostentosas, as formas escolásticas das suas teses, os préstitos, os juramentos do immaculatismo no começo dos cursos, 
consegue che, secondo Mariano Machado, "exaltados como estávamos, resolvemos, em quanto não podíamos actuar na sociedade portuguesa, reagir vigorosamente contra as praxes universitárias e a severidade do reitor, que nos submetia a uma disciplina arbitrária e injustificável"19. Il mito di Garibaldi e dei carbonari era più che mai presente quando, tra il 1861 e il 1862, Antero de Quental e alcuni altri studenti decisero di organizzarsi contro il dispotismo universitário, fondando una società segreta, la Sociedade do Raio ${ }^{20}$.

Sono queste le circostanze per cui la visita di Umberto assunse fin da subito un significato speciale. Anche in questo caso, le testimonianze sono accomunate da una parola chiave: ocasião. Per la Sociedade do Raio, la visita del principe italiano era un'occasione imperdibile per uscire allo scoperto e manifestare, allo stesso tempo, l'appoggio all'Unità d'Italia e il dissenso con l'università e con il Portogallo conservatore $^{21}$. Concretamente, il momento più propizio era il saluto

as Oraçôes de Sapiência com todo o cortejo de inesgotáveis divagações dialécticas, dão-lhe um aspecto arqueológico precioso para quem investiga as épocas históricas" (História da Universidade de Coimbra nas suas relaçôes com a instrução pública portuguesa, Lisboa, Academia Real das Ciências, 1902, vol. 4, p. 498). Altrettanto feroce il ritratto di Eça de Queiroz, che parla del rettore come "Um Czar de borla e capelo" e dell'università come una "opressão teocrática", "ultra conservadora e ultra católica", che si trasforma così in una "escola de revolução política" (Antero de Quental. In memoriam, pp. 489-490). Sul problema dell'istruzione nel Portogallo liberale: Luís Reis Torgal, A instrução pública, in História de Portugal, vol. 5, pp. 609-645.

${ }^{19}$ Antero de Quental. In memoriam, p. 150.

${ }^{20}$ António Nóvoa, rifiutando letture "folcloristiche" che sminuivano il significato della protesta della Sociedade do Raio, ne ha messo in luce aspetti organizzativi e contenuti ideologici tutt'altro che trascurabili, soprattutto se rapportati all'età dei partecipanti. Vedi: “A Sociedade do Raio na Coimbra académica de 1861-63", in Universidade(s): História - Memória - Perspectivas, Coimbra, Comissão Organizadora do Congresso "História da Universidade", 1991, vol. 3, pp. 277-320; "Em nome da liberdade, da fraternidade e da emancipação da Academia: um importante inédito de Antero de Quental redigido no âmbito das actividades da Sociedade do Raio", in Revista de História e Teoria das Ideias, 13, 1991, pp. 231-269.

${ }^{21}$ Scrive João Machado de Faria e Maia: "Em 22 de Outubro de 1862, a visita 
ufficiale a Umberto. Per piegare il saluto ai propri scopi, la Sociedade doveva ottenere un'ampia rappresentanza nella commissione incaricata di redigerlo e declamarlo. Impresa tutt'altro che facile, se si pensa che questo onore era di solito riservato agli ursos, gli studenti premiati, mentre il curriculum accademico e disciplinare di Antero e di alcuni dei suoi era pessimo $^{22}$. Tuttavia, alla cattiva reputazione si contrapponeva il grande ascendente che esercitavano su molti studenti che, seppur più moderati, dovevano condividere almeno in parte il loro disagio e avevano pertanto sposato la loro causa ${ }^{23}$. Fu così che si ebbe una commissione formata prevalentemente da studenti iscritti alla Sociedade e presieduta dallo stesso Antero $^{24}$. Soltanto una simile commissione poteva trasformare l'elogio al principe straniero nel primo atto della battaglia al rettore e a tutto ciò che rimaneva dell'ancien régime, dentro $\mathrm{e}$ fuori l'università.

Il principe Umberto arrivò a Coimbra nel pomeriggio del 21 ottobre. Anche qui, l'attendeva l'entusiasmo dei liberali:

de Humberto (actual rei de Itália) veio fornecer mais uma ocasião para um pronunciamento liberal, quer contra as ideias ultramontanas e contra o Reitor, para quem Anthero apontava, como para um phantasma do passado, ao ler o seu discurso ao príncipe, quer a favor da Itália liberta" (Antero de Quental. In memoriam, pp. 150-151). Mariano Machado de Faria e Maia: "Outra ocasião houve, em que evidenciámos os nossos sentimentos revolucionários" (ib., pp. 432-433). Teófilo Braga: "A visita do príncipe Humberto a Coimbra em 21 de outubro de 1862 deu ocasião a revelar-se esse espírito novo, que elevava esta singular geração académica" (História da Universidade de Coimbra nas suas relações com a instrução pública portuguesa, vol. 4, p. 489).

${ }^{22}$ Antero, per esempio, nel 1862-1863 ripeteva il quarto anno, e già dal primo, nell'ambito di un processo accademico, era stato segnalato come studente "muito inferior" (Mário Brandão, "Antero de Quental estudante. Documentos”, Boletim da Biblioteca da Universidade de Coimbra, 23, 1957, p. 105). Tuttavia, della Sociedade do Raio e della commissione incaricata di redigere il saluto fecero parte anche studenti brillanti come José Falcão (Fernando Catroga, "José Falcão. Um lente republicano", Biblos, 52, 1976, pp. 275-303).

${ }^{23}$ Teófilo Braga ha descritto così l'influenza del gruppo di Antero sugli altri studenti: "por esse poder, tão pouco estudado, da sugestão mútua, alguns rapazes, uma minoria que pensava e idealizava, transmitiu a sua irradiação a cérebros vulgares, 
"Coimbra vestiu-se de gala. [...] Não é ele indifferente a esta cidade ; não é para nós como qualquer outro viajante ainda que pertencente a familia real. Por um lado é ele filho do libertador da Italia [...] por outro lado é ele irmão da nossa já adorada Rainha" ${ }^{25}$. Lo stesso concetto si ritrova nei vari saluti ufficiali, accompagnato dai consueti riferimenti ai legami storici tra Italia e Portogallo. Quello della Comissão municipal, per esempio, accostava Carlo Alberto al re portoghese D. Pedro IV per la dedizione alla causa liberale, quello dell'Academia Dramática, invece, sottolineava l'affinità culturale e morale tra i due popoli, "irmãos pelas tradições gloriosas, irmãos por Colombo e Gama, irmãos por Camões e Tasso, Italianos e Portuguezes, eis-nos unidos agora em mais estreita aliança pelo enlace do Senhor D. Luís I com a Rainha a Senhora D. Maria Pia, e pelo amor às mesmas crenças" 26 .

È evidente che questi testi non brillano per originalità. Tuttavia, se accostatati al saluto di Antero, ci permettono di apprezzarne maggiormente le peculiarità e di comprendere le reazioni che suscitò a Coimbra. Per esempio, se nei primi manca qualsiasi riferimento alla figura di Garibaldi, in quello di Antero l'Italia diventa "a pátria de Garibaldi”. Poche settimane dopo la giornata dell'Aspromonte, è difficile credere

levando-os pela imitação a lerem, a pensarem, a discutir sobre interesses mentais e morais" (História da Universidade de Coimbra nas suas relaçôes com a instrução pública portuguesa, vol. 4, p. 505).

24 "Reuniu-se uma assembleia geral, no Teatro Académico, para ser nomeada a comissão, que havia de ir cumprimentar o príncipe italiano em nome da Academia. Contra a praxe de escolher só ursos (estudantes premiados) para essas comissões, conseguimos, pelas disposições que tomámos, calor e energia com que gritámos, eleger Anthero e, na quasi totalidade, rapazes do seu grupo" (Pinto Osório, Figuras do passado, Lisboa, 1815, p. 90). La commissione, oltre che da Antero de Quental, era formata da Antonio Bernardino Cerqueira Lobo, José Falcão, José de Sá Coutinho, Mariano Machado de Faria e Maia, Eduardo David e Cunha, Henrique de Macedo e José da Cunha Sampaio.

${ }^{25}$ O Tribuno Popular, 22-10-1862.

${ }^{26}$ O Tribuno Popular, 25-10-1862. 
che l'assenza di riferimenti al generale italiano sia una semplice casualità. Forse più casuale, ma non per questo meno significativo, il fatto che nel saluto di Antero non si faccia alcun riferimento a Maria Pia e al matrimonio reale. Segnali di un tono non troppo riverente, riassunto dalla frase: "Não é ao representante da casa de Saboia que vimos prestar homenagem; é ao Filho do Primeiro Soldado da Independencia Italiana" ${ }^{27}$. Con queste parole, Antero rifiutava esplicitamente il rispetto incondizionato per il principe. Questo andava guadagnato con le azioni, ed era soltanto in virtù delle azioni di Vittorio Emanuele II (e di Garibaldi) che Umberto veniva omaggiato. Teófilo Braga racconta che la frase "exerceu um prestígio enorme, difundindo em Coimbra uma atmosfera de democracia" ${ }^{28}$, e che lasciò sbigottito il principe. Scrive Mariano Machado, che faceva parte della commissione: "o príncipe perturbou-se, ruborizou-se e ficou confundido [...] era de ver a indignação que transparecia nas fisionomias de quase toda a comitiva do príncipe pelo nosso atrevimento, o qual só provocou em Cialdini e Pallavicini um mal reprimido sorriso que me pareceu muitíssimo benevolente"29. Nello stesso saluto, Antero indicava (secondo alcuni, anche con lo sguardo o addirittura con un gesto) il rettore dell'università come un fantasma do passado. Infine, la sera, nel Teatro Accademico, lo studente Fialho Machado leggeva una poesia di Antero in cui il Risorgimento diventava una parabola riv-

${ }^{27}$ Il testo completo del saluto, insieme ai nomi degli studenti in commissione, fu riportato da vari giornali, tra cui $O$ Tribuno Popular e $O$ Conimbricense del 25 Ottobre del 1862 e si trova in Antero de Quental, Prosas sócio-políticas, a cura di Joel Serrão, Imprensa Nacional, Lisboa, 1982, pp. 169-170. Sull'intero episodio, oltre alla Storia dell'Università di Teófilo Braga, è prezioso il capitolo sugli anni di Antero a Coimbra in José Bruno Carreiro, Antero de Quental. Subsidios para a sua biografia, Ponta Delgada, Instituto Cultural, 1981, 2. a ed., vol. 1.

${ }^{28}$ Teófilo Braga, História da Universidade de Coimbra nas suas relaçôes com a instrução pública portuguesa, vol. 4, p. 490.

${ }^{29}$ Antero de Quental. In memoriam, p. 433. 
oluzionaria ${ }^{30}$. La Saudação ao Principe Humberto si trasformava così nel primo atto della protesta della Sociedade do Raio. Poche settimane dopo, gli studenti abbandonavano la Sala dos Capelos durante il discorso del rettore e replicavano alle accuse con il Manifesto dos estudantes da Universidade de Coimbra à opinião ilustrada do País. La vicenda si sarebbe conclusa qualche mese dopo con le dimissioni del rettore.

Ottenuto l'obiettivo primario, la Sociedade do Raio si sciolse; tuttavia, l'episodio del saluto al principe Umberto, insieme agli altri momenti della contestazione studentesca, sarebbe rimasto a lungo nell'immaginario portoghese. Divisi da versioni discordanti, tra dimenticanze, esagerazioni e faziosità, i testimoni concordano soprattutto nell'attribuire a questo avvenimento grande importanza. Giocano un ruolo fondamentale il mito di Antero, il prestigio del principe straniero, l'orgoglio per la protesta studentesca e, ovviamente, l'immancabile saudade per gli anni da studente a Coimbra. Ancora oggi, il saluto al principe Umberto è un evento dal duplice interesse. Da una parte, è un momento decisivo nella storia dell'università di Coimbra e nella biografia di Antero de Quental, dall'altra, insieme alle nozze di Maria Pia, è uno dei tanti punti di contatto tra l'Italia del Risorgimento e il Portogallo liberale. Punti di contatto che, per la loro abbondanza, hanno già propiziato varie ricerche e probabilmente ne propizieranno molte altre.

${ }^{30}$ Anche la poesia, intitolata Itália e Portugal, fu riportata dal Conimbricense del 25 ottobre 1862. Appare, con un titolo diverso (À Itália), nella raccolta postuma Raios de extinta luz. 